\section{Vacunación contra Haemophilus influenzae tipo b en el Uruguay: experiencia e impacto}

\author{
Gloria Ruocco, ${ }^{1}$ Sergio Curto, ${ }^{2}$ \\ María Savio, ${ }^{3}$ Hilda Laurani ${ }^{4}$ \\ y Rosa Frocht ${ }^{5}$
}

\section{RESEÑA HISTÓRICA}

Uruguay tiene una larga trayectoria en materia de vacunaciones. El país ha sido uno de los primeros en la Región de las Américas en aplicar vacunas preventivas, una vez demostrada su inocuidad y eficacia en el terreno. A principios de siglo (1911) se estableció la vacunación antivariólica obligatoria y desde entonces se han ido incorporando sucesivamente nuevas vacunas: la BCG en 1927, la antidiftérica en 1944, la antipoliomielítica Salk en 1957 y la Sabin en 1962. En 1966 se instauró un programa permanente de vacunación contra siete enfermedades: viruela, tuberculosis, difteria, pertussis, tétanos, poliomielitis y sarampión. En 1982 se estableció en el país el Programa Ampliado de Inmunización (PAI) de alcance nacional, en el cual tienen que participar obligatoriamente todos los ciudadanos según lo estipula el decreto ley 15.272. En el PAI se incluían entonces las vacunas BCG (antituberculosa), DPT (contra difteria, pertussis y tétanos), antipoliomielítica y triple viral SRP (contra sarampión, rubéola y parotiditis).

El Ministerio de Salud Pública, por conducto de la División de Epidemiología y su Sección de Inmunizaciones, adquiere las vacunas y las ofrece gratuitamente a la población. A partir de 1987 se incorporó un nuevo actor al programa, la Comisión Honoraria para la Lucha Antituberculosa y Enfermedades Prevalentes, que tiene la responsabilidad de distribuir las vacunas y ejecutar el programa. La vacunación se realiza de forma individualizada mediante un registro computadorizado que permite vigilar el estado vacunal de cada niño que nace en el país, identificar a los niños que no están al día con el cronograma de vacunación y a la vez obtener datos más confiables sobre la cobertura por cohorte. En 1994 se añadió a los ocho preparados inmunobiológicos usados en el programa la vacuna contra Haemophilus influenzae tipo b (Hib).

\section{Meningitis bacterianas}

Uruguay, Ministerio de Salud Pública, División de Epidemiología. Dirección postal: 18 de julio 1892, Montevideo, Uruguay.

2 Uruguay, Ministerio de Salud Pública, Programa Ampliado de Inmunización y Departamento de Vigilancia Epidemiológica.

3 Uruguay, Ministerio de Salud Pública, Sección de Enfermedades Transmisibles.

4 Comisión Honoraria para la Lucha Antituberculosa y Enfermedades Prevalentes, Departamento de Vacunaciones.

5 Comisión Honoraria para la Lucha Antituberculosa y Enfermedades Prevalentes, Laboratorio "A. Calmette".

\footnotetext{
La División de Epidemiología del Ministerio de Salud Pública y la División de Laboratorios de Salud Pública han mantenido desde 1979 una red de vigilancia de las meningitis supurativas en todo el país. Esta vigilancia consiste en recibir las notificaciones de los casos de acuerdo con lo establecido
} 
FIGURA 1. Casos por 100000 habitantes de meningitis supurativa neumocócica, meningocócica y por Haemophilus influenzae tipo b (Hib) durante el período anterior a la introducción de la vacuna contra Hib, Uruguay, 1979 a 1994

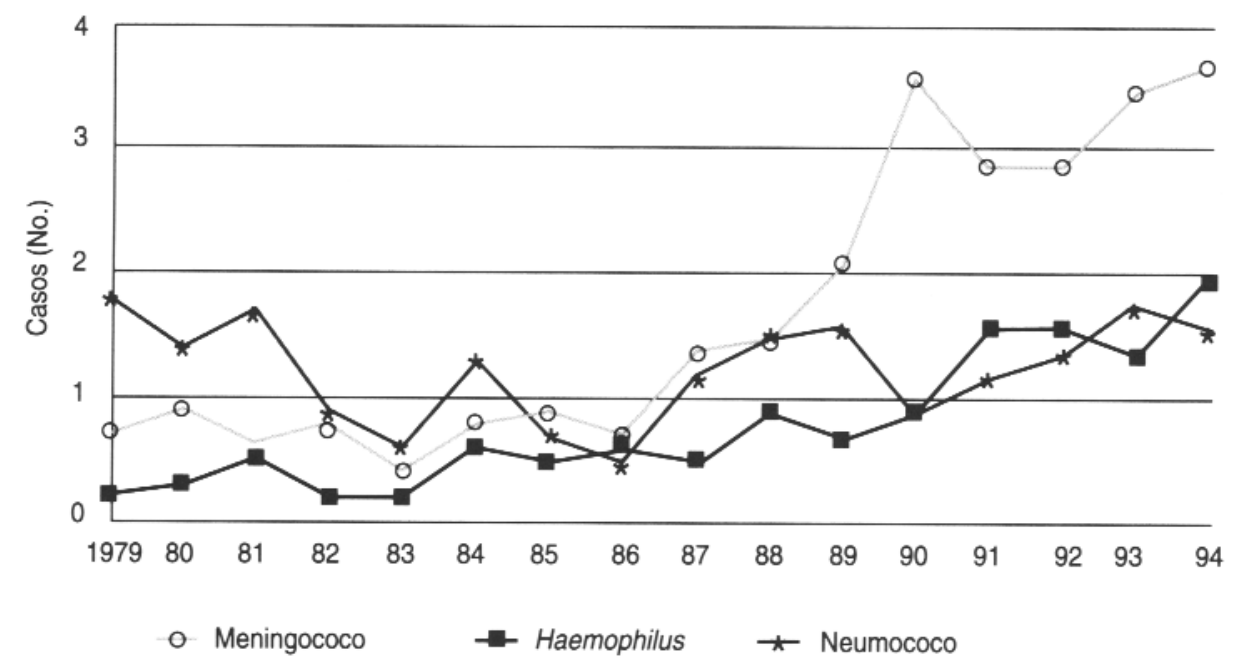

por la norma legal vigente sobre enfermedades de notificación obligatoria, la búsqueda activa de casos en los servicios asistenciales públicos y privados, y la investigación epidemiológica de cada caso con su correspondiente seguimiento y control y sus contactos.

En los laboratorios de los hospitales del Uruguay se hace todo lo posible por identificar el agente causal de cada caso y, si eso no es posible, se envían los especímenes correspondientes al laboratorio central. Para 1987 esta vigilancia mostraba un aumento progresivo de las meningitis debido en parte al mejoramiento del sistema de vigilancia y en parte a la mejor capacidad diagnóstica de los laboratorios. Mientras que el número de casos por Streptococcus pneumoniae se mantenía constante, se observaba un aumento de las meningitis por Neisseria meningitidis y H. influenzae, como puede verse en la figura 1. Para 1993, la meningitis meningocócica había alcanzado una incidencia de 3,5 por 100000 habitantes con una letalidad de $15,4 \%$ en la población general, mientras que la meningitis por Hib alcanzaba 1,5 por 100000 (15,6 por 100000 en los menores de 5 años) con una letalidad de 9,1\%. El grupo de edad más afectado por las meningitis correspondía a los menores de 5 años y en ese grupo de edad se registraban $58 \%$ de los casos de meningitis meningocócica y $97 \%$ de los casos de meningitis por Hib. Sin embargo, $70 \%$ de estos últimos estaban concentrados en el grupo de 1 a 11 meses de edad.

\section{Vacunación contra Haemophilus influenzae tipo b}

Frente a la situación epidemiológica manifestada en 1994 y teniendo en cuenta la existencia de una vacuna sumamente eficaz, el Ministerio de Salud Pública resolvió incorporarla al cronograma de vacunaciones obligatorias para los menores de 5 años, lo que se hizo efectivo por medio de un decreto del poder ejecutivo. La vacunación ha sido gratuita desde el principio. El esquema de aplicación de la vacuna se ajustó de acuerdo con la edad:

a) los niños de 2 meses recibirían tres dosis a los 2 , 4 y 6 meses de edad, con una dosis de refuerzo entre los 12 y 15 meses;

b) entre los 7 y 11 meses de edad, se aplicarían dos dosis con un intervalo entre sí de 2 meses y una dosis de refuerzo entre los 12 y 15 meses, y

c) los niños de 1 a 4 años recibirían una dosis única.

En el período de 4 meses comprendido entre el 29 de agosto y el 31 de diciembre de 1994, de un total de 257000 niños de 2 meses a 4 años de edad, se vacunaron 196843 que correspondieron a $76,6 \%$ de dicha población.

La figura 2 muestra el número de casos mensuales de meningitis debidas a Hib desde enero de 1993 hasta julio de 1997. Es importante destacar el extraordinario impacto logrado por la vacunación y en especial la rápida disminución de los casos en 
FIGURA 2. Incidencia de meningitis por Haemophilus influenzae tipo b (Hib), Uruguay, desde enero de 1993 hasta julio de 1997

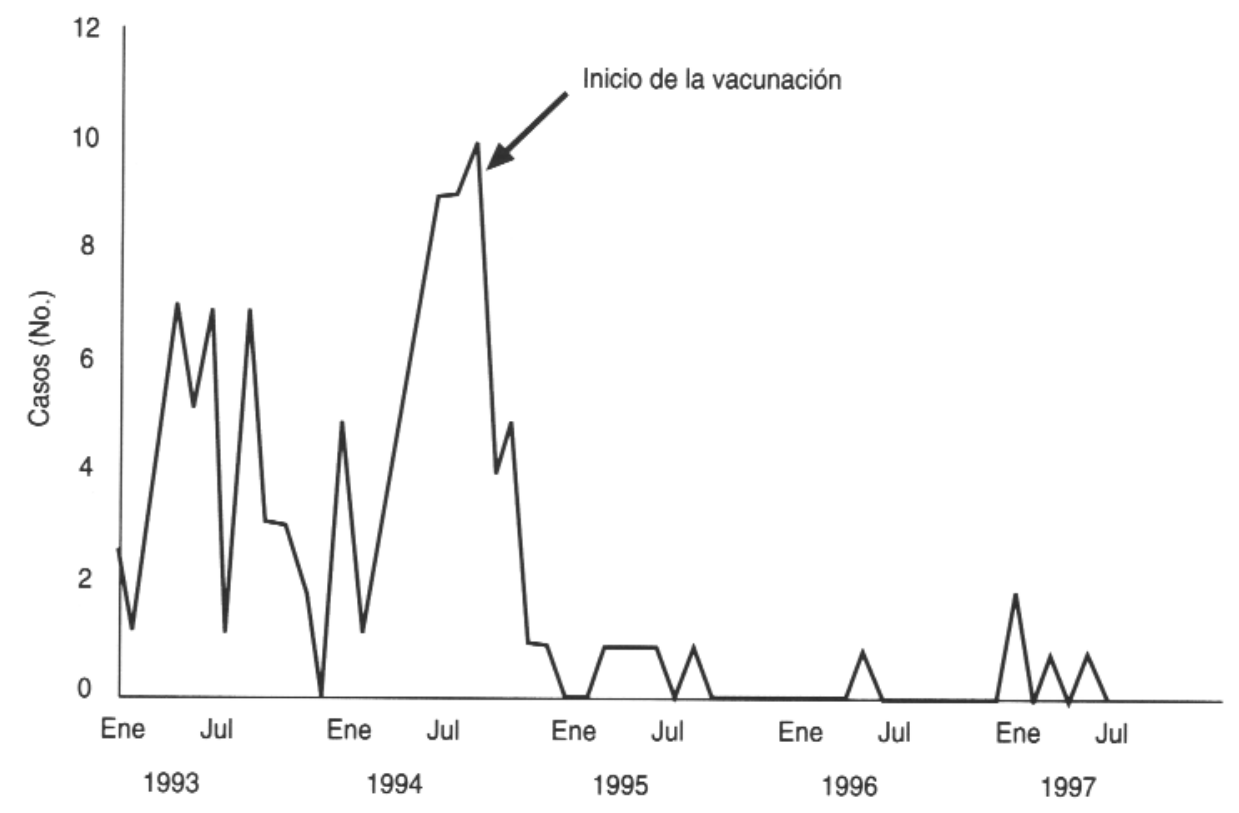

niños menores de 4 años que se alcanzó en los primeros 6 meses después de la introducción de la vacuna. Desde entonces hasta marzo de 1988 se observaron solamente 10 casos de meningitis por Hib en todo el país. El análisis de la información disponible indica que de esos casos, ocho no estaban inmunizados y tres correspondían a grupos de edad no incluidos dentro del esquema de vacunación $(6,17$ y 31 años, respectivamente). De los inmunizados, uno había recibido una sola dosis y el otro había completado el esquema completo de las tres primeras dosis.

Es importante destacar que desde 1993 se viene realizando un estudio de vigilancia epidemiológica de Streptococcus pneumoniae en niños menores de 5 años con enfermedades invasoras (neumonía, meningitis, sepsis, etc.) y se puede observar la desaparición de $H$. influenzae tipo b como agente causal de las infecciones respiratorias. Estudios previos indicaban un aislamiento de este agente patógeno en aproximadamente $2,2 \%$ de las neumonías.

\section{Conclusiones}

Desde que se incorporó en el programa regular de inmunización del Uruguay el uso de una vacuna segura y efectiva contra $H$. influenzae tipo b, se ha visto su enorme impacto en el control de las enfermedades provocadas por ese agente patógeno. Este es un ejemplo más, si bien fue el primero en la Región, de los efectos que esta vacuna puede tener como intervención de salud pública.

\section{SYNOPSIS}

\section{Vaccination against Haemophilus influenzae type $b$ in Uruguay: experience and impact}

Between 1979 and 1994, epidemiological surveillance of meningitides in Uruguay showed a progressive increase in suppurative meningitides due mainly to Neisseria meningitidis and Haemophilus influenzae type $b$ (Hib). The cases were concentrated in children under 5; however, among the cases caused by Hib, 70\% affected children from 1 to 11 months old. Facing this situation, the Ministry of Public Health resolved, as of August 1994, to include the Hib vaccine in the country's Expanded Program on Immunization, which has been in place since 1982.

The Hib vaccination is done without charge and is obligatory for all children under 5 years of age. It is done using the following series of vaccinations: a) three doses, given at 2,4 , and 6 months, with a booster dose at age $1 ; b$ ) children from 7 to 11 months old receive two doses two months apart and a booster dose a year later; and c) a single dose for children 12 months to 4 years old. Between August and December 1994 a coverage rate of $76.6 \%$ was reached among children between 2 months and 4 years old, and the coverage has remained above $80 \%$ in the new cohorts.

In Uruguay, this vaccination strategy had a spectacular impact on morbidity and mortality due to meningitides caused by Hib. One of the results was that the incidence of 15.6 per 100000 registered in children under 5 in the prevaccination years declined to 0.03 per 100000 in 1996. 